ENCYCLOPEDEE Encyclopédie berbère

BERBERE

$11 \mid 1992$

11 | Bracelets - Caprarienses

\title{
Bronze (Âge du)
}

voir Campaniforme - Cuivre (Âge du)

G. Camps

\section{OpenEdition}

\section{Journals}

Édition électronique

URL : http://journals.openedition.org/encyclopedieberbere/1872

DOI : $10.4000 /$ encyclopedieberbere.1872

ISSN : 2262-7197

\section{Éditeur}

Peeters Publishers

\section{Édition imprimée}

Date de publication : 1 septembre 1992

Pagination : 1614-1626

ISBN : 2-85744-581-4

ISSN : $1015-7344$

\section{Référence électronique}

G. Camps, «Bronze (Âge du) », Encyclopédie berbère [En ligne], 11 | 1992, document B108, mis en ligne le 01 juin 2012, consulté le 24 septembre 2020. URL : http://journals.openedition.org/ encyclopedieberbere/1872 ; DOI : https://doi.org/10.4000/encyclopedieberbere.1872

Ce document a été généré automatiquement le 24 septembre 2020.

(c) Tous droits réservés 


\section{Bronze (Âge du)}

voir Campaniforme - Cuivre (Âge du)

\section{G. Camps}

1 En un siècle et demi de recherche archéologique, l'Afrique du Nord a livré moins d'une trentaine d'armes ou d'instruments en cuivre ou en bronze qui soit parvenue à la connaissance des spécialistes.

2 Comment expliquer cette carence? La rareté des objets métalliques attribuables au Chalcolithique ou à l'Âge du bronze demeure l'un des problèmes non résolus de la Protohistoire maghrébine. 


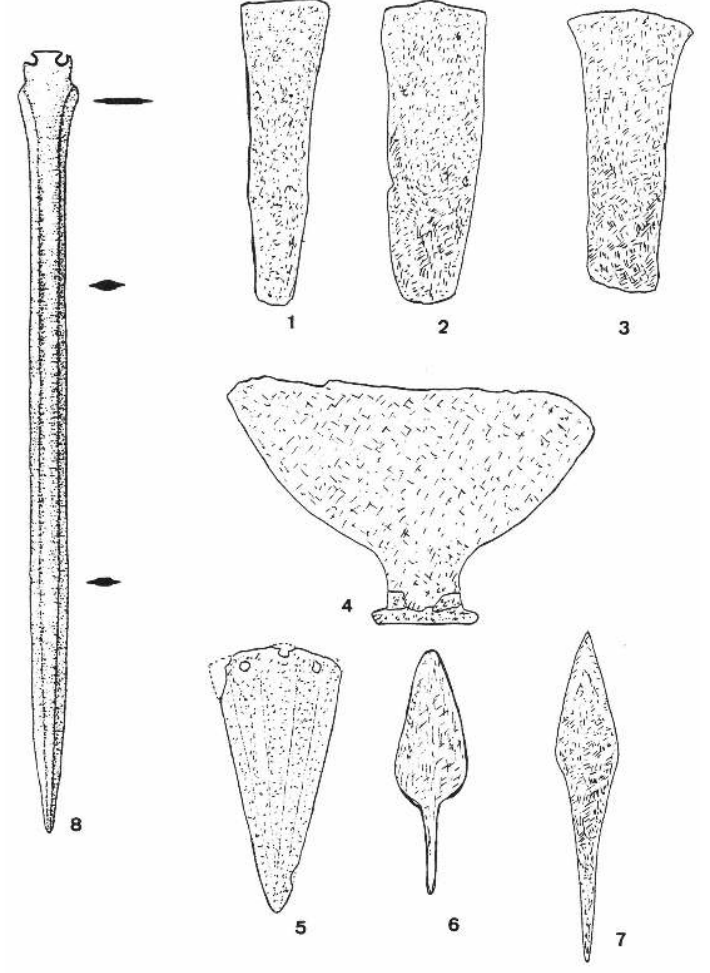

1. Hache de l'oued Akrech ; 2 . Hache en cuivre du Kef el Baroud ; 3 . Hache en bronze de Columnata ; 4. Hache de l'oued Kniss, Beni Snassen ; 5. Hallebarde de Mers ; 6. Pointe de Palméla de Sidi Messaoud ; 7. Pointe de Palméla d'Aïn Dahlia ; 8. Epée de bronze de Larache. Les objets sont représentés à des échelles différentes.

Ce ne peut être, en effet, la rareté des gisements de cuivre qui puisse l'expliquer; le Maghreb possède un nombre considérable de gisements. Certes, mis à part quelques gisements majeurs qui sont seuls exploités aujourd'hui, la plupart ne sont que de simples gîtes métallifères, dont l'exploitation n'est pas recommandée dans une économie moderne fondée sur la rentabilité, mais qui durant l'Antiquité et la Préhistoire étaient susceptibles d'alimenter un artisanat de valeur. L'étain est beaucoup plus rare, bien qu'un gisement d'une certaine importance, celui d'Oulmès, au Maroc, ait été exploité à l'époque moderne. Mais l'exemple de maintes régions européennes montre que l'existence d'une industrie du bronze n'est pas nécessairement associée à une exploitation locale de l'étain. Sur le plan minéralogique le Maghreb n'est donc pas plus mal desservi que l'Europe ; un âge des métaux aurait pu s'y développer aussi bien qu'au nord de la Méditerranée.

\section{À la recherche d'un Âge du bronze}

Aussi peut-on se demander si la pauvreté de notre documentation sur les armes et objets de bronze en Afrique du Nord n'est pas simplement due à la faiblesse de l'exploration archéologique.

Il suffit de remarquer combien l'action d'un homme, telle celle de J. Malhomme pour la découverte et la publication des représentations d'armes en métal dans le Haut Atlas marocain, peut être déterminante et agrandir brusquement le champ de la 
connaissance. Inversement plusieurs exemples douloureux révèlent la négligence de préhistoriens chevronnés à l'égard de documents en métal: ce sont soit des descriptions incomplètes, soit des pertes d'objets, soit enfin le mutisme total. Une part importante des découvertes peut ainsi avoir échappé à la connaissance des rares spécialistes.

6 Une autre raison, qui peut ne pas être facilement reçue dans un pays riche et fortement industrialisé mais qui ne saurait être négligée dans un pays pauvre, est l'habituel souci de récupération qui fait recueillir le moindre objet de métal pour l'utiliser tel quel ou le refondre. Pour illustrer cet état d'esprit nous rappellerons seulement l'étonnement des officiers français lorsqu'ils constatèrent, au moment de l'occupation de Tébessa (Algérie orientale) que les monnaies romaines étaient utilisées dans les transactions, encore au milieu du xix siècle.

Quelle que soit la validité des différentes raisons que nous avons exposées, il n'en demeure pas moins que les instruments et les armes en cuivre et en bronze sont d'une très grande rareté au Maghreb mais moins sans doute qu'on ne le pensait, ou disait. Cette rareté ne justifie pas cependant la position négative qui fut longtemps celle des Préhistoriens quant à l'existence d'un Âge du bronze en Afrique du Nord. P. Pallary dans le premier quart du siècle n'était pas loin de croire que ce qu'il appelait le Néolithique berbère (qui, en fait, est une industrie paléolithique que nous nommons l'Atérien) florissait encore aux temps de l'Empire romain. De son côté S. Gsell, le grand historien de l'Afrique du Nord, estimait, au même moment, que « le cuivre et le bronze aient été très peu répandus parmi les indigènes, ou ignorés d'eux, avant l'époque où ils commencèrent à faire usage du fer. Chez les populations voisines du littoral, des objets en métal durent être introduits par des étrangers, surtout par les marchands des colonies maritimes phéniciennes qui furent fondées à partir de la fin du second millénaire ».

8 Comme il arrive bien souvent, cette simple hypothèse de travail est passée pour une vérité démontrée et chacun de répéter qu'il n'y a pas d'Âge du bronze en Afrique du Nord. Du même coup se manifestait une tendance très nette à mettre en doute l'authenticité des trop rares documents reconnus, ainsi G. Souville a peine à croire que le Dr Bourjot ait trouvé dans son jardin, à Saint-Eugène près d'Alger, en hache à talon, que E. Pelagaud remit ensuite au Musée de Lyon. C'est peut-être le Dr Gobert qui alla le plus loin dans cette position négative puisqu'il crut, au moins pendant un certain temps, à la coexistence de peuplades restées à l'état prénéolithique et des premiers colons phéniciens. 


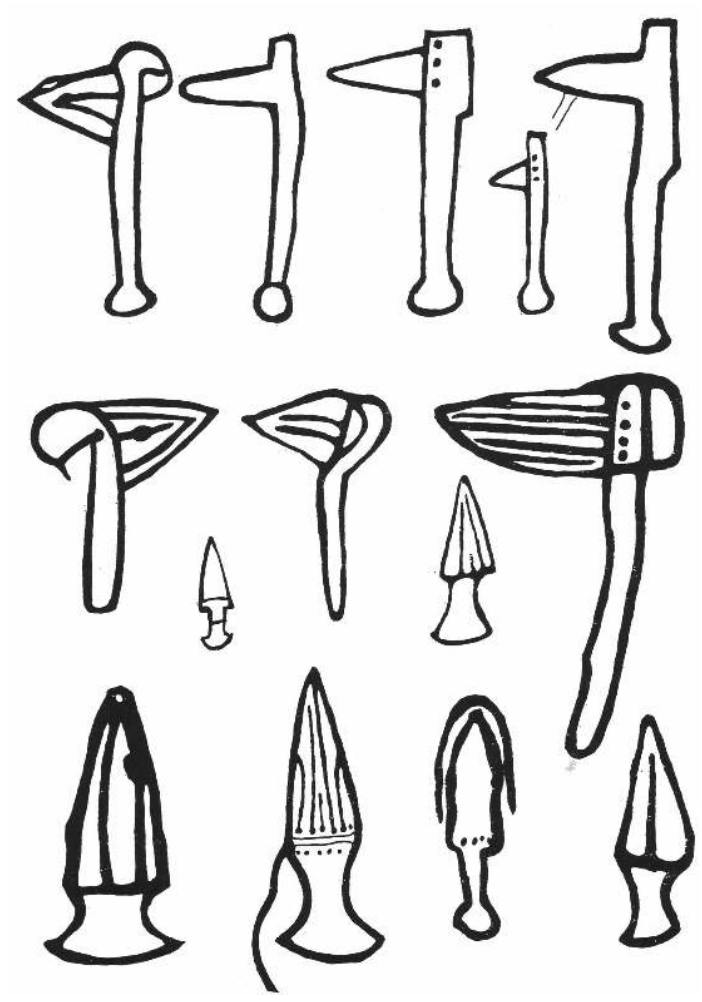

9 Telle était la situation vers 1955. On connaissait bien quelques objets en métal mais la plupart n'avaient pas été trouvés dans des conditions archéologiquement satisfaisantes; leur publication était insuffisante et l'idée dominait que les Berbères étaient restés à l'Âge de la pierre jusqu'à l'arrivée des Phéniciens qui leur auraient directement enseigné l'usage et le travail du fer.

Cette position n'aurait pu cependant résister à un examen attentif des données archéologiques. On y reconnaît, au départ, une grave erreur méthodologique qui consiste à ne retenir que les seuls objets métalliques et à oublier l'existence d'autres documents aussi caractéristiques tels que les céramiques, ou de négliger la pénétration de types de sépultures européennes tels que les dolmens et les hypogées, antérieurement à toute navigation orientale d'âge historique. Il y avait des relations suivies entre le Maghreb et l'Europe à l'Âge du bronze, elles faisaient suite à celles qui existaient au Chalcolithique et tout au long du Néolithique. L'Afrique du Nord n'était donc ni isolée ni fermée à toute innovation.

Un autre argument qu'auraient pu faire valoir les partisans d'un Âge du bronze nordafricain était la connaissance que l'on avait, grâce aux Egyptiens, de ces Libyens orientaux, Mashaouash, Lebou, Tehennou et autres tribus qui, maintes fois, s'attaquèrent à l'Egypte. Or, en plein Âge du bronze, au XIII ${ }^{\mathrm{e}}$ siècle avant J.-C. nous apprenons, par exemple, par l'inscription de Karnak en l'honneur de la victoire remportée par Mineptah sur les Mashaouash qu'ils abandonnèrent aux armées du pharaon, 9111 glaives en bronze. Ce chiffre, dont la précision ne trompe guère, est vraisemblablement exagéré, mais il témoigne de la connaissance, et certainement du travail du métal chez les Libyens orientaux. Pourquoi leurs frères d'Occident, en 
relation avec des pays qui, tout comme l'Egypte, avaient de solides traditions métallurgiques, seraient-ils restés dans un âge primitif de la pierre?

Une révision des documents connus et l'exploitation de nouvelles découvertes devaient conduire l'auteur de cette notice à renverser la tendance et à se prononcer en faveur de l'existence d'un Chalcolithique et d'un Âge du bronze en Afrique du Nord.

\section{Chalcolithique et Âge du bronze au Maroc}

Cette révision avait été préparée par la découverte ou la publication correcte de nouvelles armes en cuivre comme le poignard du Chenoua près de Tipasa à l'est d'Alger, ou en bronze comme des haches de Columnata dans la région de Tiaret et de l'Oued Akrech près de Rabat. Plus intéressante encore était la mise en lumière par J. Malhomme d'un extraordinaire ensemble de gravures rupestres dans le Haut-Atlas marocain, très différentes des œuvres néolithiques de l'Atlas saharien et de l'Anti-Atlas. Cet ensemble compte de nombreuses représentations d'armes en métal fort reconnaissables : ce sont des poignards à rivets et à nervures connus en Europe dès le Bronze ancien, des hallebardes, armes très caractéristiques dont la lame de même forme que celles des poignards était renforcée et fixée perpendiculairement à un manche, d'où le nom de haches-poignards que leur avait donné J. Déchelette. Les hallebardes apparaissent au Bronze ancien et sont particulièrement nombreuses en Espagne (culture d'El Argar). Ces représentations, très méridionales, permettaient d'entrevoir l'importance de la pénétration argarique au Maroc. Mais, antérieurement, d'autres relations s'étaient déjà nouées avec la Péninsule ibérique. Elles sont prouvées par la découverte de vases campaniformes* sur le littoral marocain, depuis le détroit de Gibraltar jusque dans la région de Casablanca. En Oranie un seul tesson était connu dans la grotte de Rhar Oum el Fernan (Saïda), un second fut reconnu dans les collections du Musée d'Oran provenant de l'Oued Saïda.

Depuis 1960, durant les trois dernières décennies, de nouveaux documents de grand intérêt sont venus à notre connaissance et confirment, en les précisant les conclusions précédentes.

Le Maroc du Nord s'est, une fois encore, révélé plus riche que les autres régions. Il faut noter en effet la redécouverte et la publication de la pointe trouvée par G. Buchet dans une tombe en ciste d'Aïn Dalia (El Mriès) dans la région de Tanger, qui n'était connue que par une description incomplète. Suivant cette description, cette arme pouvait être considérée aussi bien comme une pointe de lance ou un poignard à languette. C'est en réalité une pointe en cuivre à limbe triangulaire allongé muni d'une soie. Il s'agit d'une variété des pointes du type de Palmela. Ces pointes d'origine ibérique se sont répandues pendant le Chalcolithique et le Bronze ancien dans les régions atlantiques et les pays méditerranéens occidentaux. La pointe d'Aïn Dalia n'est connue que par une photographie conservée au Musée de l'Homme à Paris, l'objet a disparu ainsi que l'alène (l'inventeur disait un poinçon) découverte dans le même ciste.

Plus intéressante encore est la découverte par J. Ponsich dans une autre tombe en ciste de la région (nécropole de Mers), d'une arme qualifiée de «lame de poignard ou de hallebarde en bronze». Malgré ses faibles dimensions $(10,5 \mathrm{~cm}$ de long et $5 \mathrm{~cm}$ de largeur maximum), H. Schubart et R. Chenorkian estiment qu'il s'agit d'une hallebarde, tant en raison de sa silhouette que de la présence d'une forte nervure. Les dimensions réduites de cette arme s'expliquent peut-être par le fait qu'ayant été trouvée dans une 
tombe, elle n'avait pas de caractère fonctionnel. Par sa morphologie, la hallebarde de Mers appartient au type ibérique de Carrapatas dont elle possède tous les caractères: nervure, cannelures, extrémités proximale à trois rivets. La même sépulture contenait deux alènes.

Deux autres documents d'intérêt moindre méritent d'être rappelés bien que leur origine soit douteuse. G. Souville a signalé une hache plate dans les collections du Musée de Tétouan, semblable à celle de l'Oued Akrech, qui est peut-être celle par C. de Montalban à Arzila. Une autre hache de grandes dimensions $(18,2 \mathrm{~cm}$ de long et $28,8 \mathrm{~cm}$ de large) provient des Monts des Beni Snassen, peut-être des gorges de l'Oued Kiss. Elle est aujourd'hui déposée au Musée de Rabat. Sa forme, rare, est celle d'un éventail faiblement dissymétrique ; le talon est emboîté dans une pièce en bronze qui fut brasée sans doute à une époque plus récente. Les analyses chimiques indiquent 7 à $8 \%$ d'étain et des traces de plomb. Avec de grandes précautions G. Souville propose de rapprocher cette hache de celles en forme de pelte représentées dans les gravures rupestres de l'Oukaïmeden (Haut-Atlas) et sur certaines stèles portugaises.

Le Maroc atlantique a apporté de nouveaux témoignages de l'expansion du vase campaniforme : deux poteries entières, provenant vraisemblablement d'une sépulture, ont été mises au jour à Sidi Slimane du Rharb et publiées par G. Souville. Ces vases sont parfaitement identifiables, ils appartiennent au groupe portugais du style campaniforme. De nouveaux témoignages ont été trouvés dans la région de Fès.

La grotte du Kef el Baroud dans la région de Casablanca a livré trois objets de métal qui présentent un grand intérêt; il s'agit d'une hache plate en cuivre et de deux grandes alènes. La hache et l'une des alènes furent recueillies dans les déblais, mais la seconde alène provient de la couche grise qui fut datée par C 14 de $5160 \pm 110$ ans soit 3210 BC. Cette datation paraît un peu haute, d'autant plus que la couche blanche qui lui est antérieure d'après la stratigraphie accuse un âge de $4750 \pm 110$ ans soit $2800 \mathrm{BC}$. Les deux objets trouvés hors stratigraphie ne doivent pas être négligés ; pour A. de Wailly ils proviennent de la couche grise puisqu'ils ont été recueillis dans une poche dont le toit était constitué de matière de remplissage de cette couche. Les conditions nous paraissent suffisantes pour penser que le cuivre était connu au Maroc au moins au début $\mathrm{du} \mathrm{III}^{\mathrm{e}}$ millénaire. Cette découverte, faite malheureusement dans des conditions qui ne sont pas parfaites, est la plus importante de ces dernières années. 
Hache de l'oued Akrech. Longueur 15,5 cm. Pointes de Palméla de Karrouba et de la Stidia encadrant la hache de Karrouba. Longueur de la hache 9,5 cm.
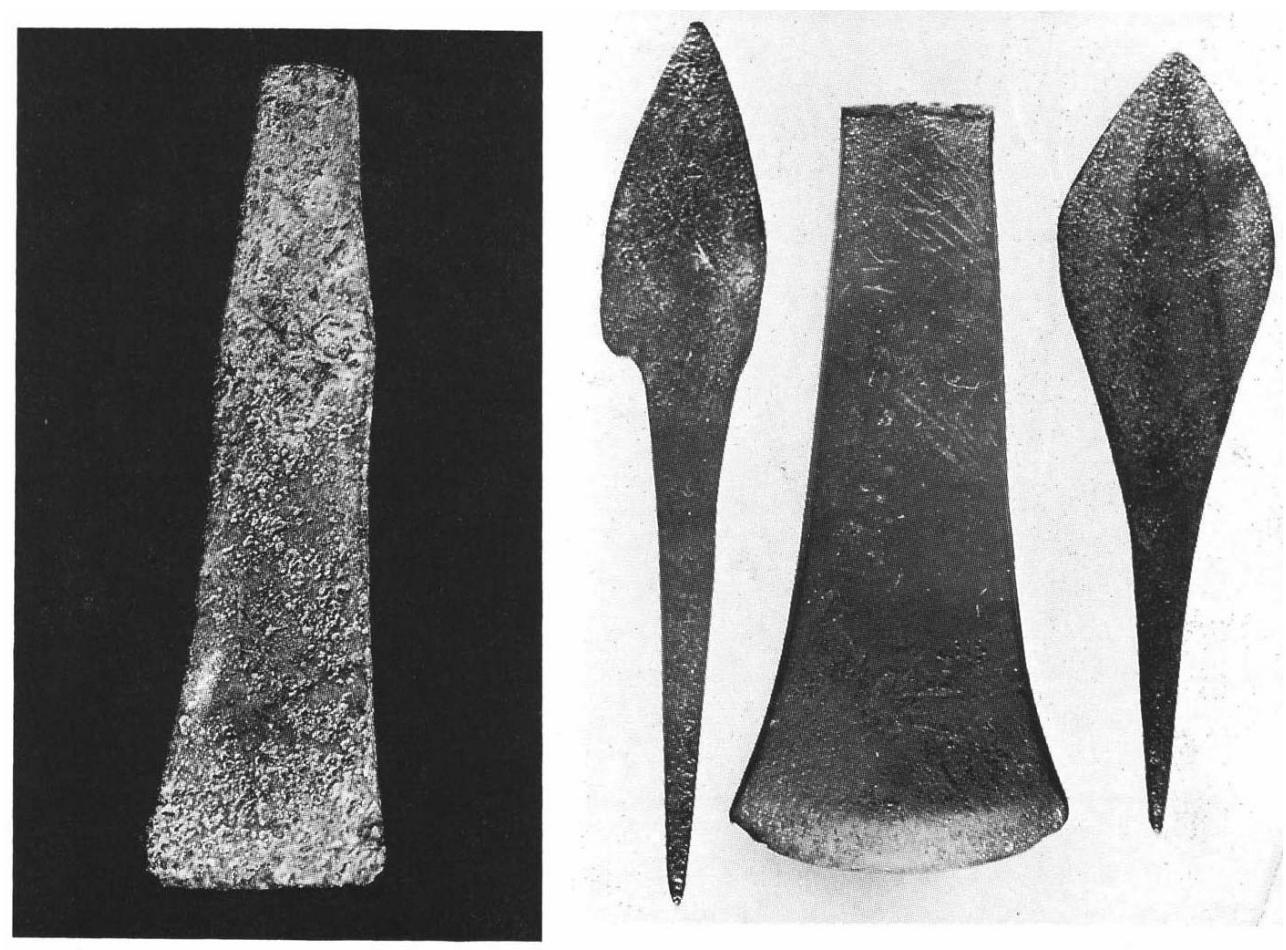

Il importe également de signaler la découverte par L. Wengler d'une nouvelle pointe de Palméla dans la grotte d'El Hariga (Maroc oriental) et d'une autre à Aïn Smène, dans la région de Fès.

Aux nombreuses représentations d'armes métalliques publiées entre 1950 et 1960 dans le Haut Atlas par J. Malhomme, particulièrement celles des stations de l'Oukaïmeden et du Yagour, il faut ajouter de nombreuses autres figurations signalées par A. Simoneau dans le massif du Rat ou dans l'Aougdal n'Oumghar ainsi que dans l'Anti-Atlas; mais c'est à R. Chenorkian que nous devons l'étude la plus complète des armes* en métal figurées sur les parois rocheuses de l'Atlas marocain. En plus des boucliers, nommés « disques » par J. Malhomme, et des arcs rares, on reconnaît de nombreux poignards, des hallebardes, différentes formes de haches et des "pointes", dont le manche très court, semblable à celui des armes plus récentes qui figurent sur des monuments d'âge historique ou qui sont citées par les auteurs antiques, incite à les considérer comme des sagaies plutôt que comme des lances ou des flèches. R. Chenorkian reconnaît, parmi les quelque 300 représentations de poignards, trois types dont le premier possède une garde cruciforme (Type I a) ou faiblement saillante (Type Ib). Les types II et III ont des poignées plus étroites, dépourvues de garde, avec ou sans pommeau; comme sur les poignards à rivets ou à manche massif de l'Europe méridional, il est possible de reconnaître des lames à filets gravés parallèles aux bords ou à nervure axiale.

Plus intéressante encore est l'analyse portant sur une quarantaine de figurations de hallebardes, parmi lesquelles R. Chenorkian reconnaît trois types; le premier est comparable aux hallebardes ibériques du style d'El Argar à lame étroite présentant un emmanchement robuste grâce à l'élargissement de la partie proximale de la lame. $R$. Chenorkian remarque sur les figurations atlasiques un renflement important à la partie distale du manche, sans doute pour augmenter le poids de l'arme à son extrémité et la 
rendre plus efficace. Il pense justement que ce renflement qui s'étend parfois jusqu'à la région médiane du manche s'imposait en raison de la forme et des dimensions de la plaque de fixation des lames, telles que le montrent les exemplaires métalliques découverts dans la Péninsule ibérique. Le type suivant se subdivise en II a et II $b$ d'après la place de la lame sur le manche. Les lames de ces hallebardes sont larges, de forme triangulaire, comme celles du type de Carrapatas, en Espagne. Elles sont renforcées par une nervure. La petite hallebarde, peut-être votive, trouvée dans la sépulture de Mers (région de Tanger) appartient à ce type. Les hallebardes du troisième type ont une lame moins large mais plus longue, le manche présente parfois un crochet externe à l'extrémité distale et un renflement proximal ; elles n'ont pas leur équivalent dans la Péninsule ibérique.

Parmi les haches représentées dans l'Atlas marocain, une forme particulière mérite de retenir l'attention. La hache-pelte est également figurée dans le Sahara occidental (Méran) et présente une grande ressemblance avec les «haches-idoles » des stèles ibériques étudiées par M. Almagro.

Dans le corpus des gravures rupestres de l'Atlas, il n'y a aucune représentation d'épée pouvant remonter à l'Âge du bronze mais il nous faut citer la belle épée trouvée dans l'estuaire du Loukkos (Larache). Cette arme, signalée rapidement par B. Saez Martin, en 1952, fut longtemps considérée comme perdue; elle a été récemment retrouvée au Musée de Berlin et publiée. Il s'agit d'une lame aux bords parallèles appartenant sans discussion au complexe du Bronze atlantique.

Aux objets et armes diverses en métal qui sont des témoins principaux de l'existence d'un Âge du bronze dans l'ouest du Maghreb, il importe d'ajouter quelques traits culturels qui, sans être aussi précis, n'en contribuent pas moins à enrichir la documentation. Nous devons, en premier lieu, retenir certains types de sépulture qui, indépendamment de leurs caractéristiques ayant valeur chronologique, présentent une localisation étroite se confondant avec la zone d'expansion des influences ibériques qui sont à l'origine de l'Âge du bronze maghrébin. Si les sépultures en jarres (ces dernières non décrites) de Zemamra près de Mazagan se rattachent peut être à la culture d'El Argar (Bronze ancien ibérique), on est plus assuré du caractère protohistorique des tombes en ciste, longtemps confondues avec des dolmens, des nombreuses petites nécropoles de la région de Tanger, la forme des monuments, leur localisation et le mobilier qui y fut trouvé (hallebarde de Mers, pointe de Palméla d'Ain Dahlia) sont autant de témoins de leur appartenance à l'Âge du bronze. Les tombes en forme de silo, localisées dans le Maroc oriental et en Oranie, ont été rapprochées des sépultures de forme semblable du Chalcolithique et du Bronze ancien du Portugal et d'Andalousie.

La céramique de l'Âge du bronze commence à être mieux connue dans les divers gisements du Maroc septentrional. C'est une céramique à fond plat, grise, noirâtre ou rouge, ayant dans ce cas subi un polissage intense, qui apparaît au-dessus des niveaux à céramique campaniforme*. Malheureusement, trouvée dans des niveaux superficiels et d'aspect grossier, elle fut souvent négligée ou considérée comme récente.

Le Maroc a également livré d'autres monuments difficiles à dater et qui sont habituellement, pour des raisons uniquement stylistiques, attribués au début de l'Âge des métaux. Ce sont trois stèles ornées, deux à N'Kheila, une à Maaziz. La mieux conservée (N'Kheila I) est en grès, elle présente une figure humaine, nue semble-t-il, à tête discoïde ; cette figure est entourée de demi-cercles concentriques et d'arceaux qui sont d'aspect atlantique et rappellent les décors des pétroglyphes de Bretagne et 
d'Irlande. L'autre stèle de N'Kheila présente les mêmes motifs entourant un anthropomorphe en grande partie disparu. La stèle de Maaziz présente un décor qui n'est pas sans analogie avec celui des stèles chalcolithiques des Alpes italiennes.

\section{Les traces d'un Âge du bronze en Algérie occidentale}

L'Algérie fut toujours plus pauvre en objets de métal que le Maroc atlantique et septentrional. Assez bizarrement c'est la région comprise entre Alger et Cherchel qui jusqu'en 1960 avait fait connaître de rares documents dont le plus ancien est sans conteste le poignard en cuivre du Chenoua. Près de Tiaret, P. Cadenat avait découvert en 1955, à Columnata, dans un labour, une hache plate en bronze contenant $7,60 \%$ d'étain. Quelques années plus tard, il trouvait dans la Vigne Serrero, dans la même région, une petite pièce de cuivre ou de bronze qui semble appartenir à une pointe de Palméla de petites dimensions et divers autres fragments, dont un poinçon en bronze.

Plus intéressante et inattendue fut la redécouverte, par le même chercheur dans les collections du Musée de Figeac (Lot, France), de trois pièces provenant de la région de Mostaganem (Algérie). Ces objets ont été trouvés, on ne sait dans quelles circonstances, par M. Bouyssou qui était agent voyer à Mostaganem avant 1934. Deux de ces pièces proviennent de Karrouba à $4 \mathrm{~km}$ au nord-est de Mostaganem. Il s'agit d'une hache et d'une pointe. La hache a de très faibles rebords à peine marqués, et un tranchant concave qui permettent de la classer dans le type de Neyruz ou de La Polada qui est très répandu au Bronze ancien en Suisse, dans l'est de la France et en Italie. Longue de 9,1 $\mathrm{cm}$, cette hache a un talon relativement étroit $(1,7 \mathrm{~cm})$ et légèrement martelé. Le tranchant modérément convexe a une corde de $4 \mathrm{~cm}$. L'autre pièce provenant de Karrouba est une pointe du type de Palméla, la dissymétrie du limbe est peut-être due à la disparition d'un aileron. La largeur maximum est actuellement de 1,8 cm, la longueur de $10 \mathrm{~cm}$.

Le Musée de Figeac possède également une seconde pointe du type de Palméla recueillie par M. Bouyssou à La Stidia, village littoral situé à l'ouest de Mostaganem. Plus courte que la précédente $(7,7 \mathrm{~cm})$, elle possède un limbe large et peu acéré. Comme sur celle d'Aïn Dalia (Tanger) les bords ont été martelés, laissant en relief un triangle nettement délimité qui occupe l'axe de la pièce.

\section{Monuments mégalithiques et hypogées d'Algérie orientale et de Tunisie}

Fait curieux, alors que les témoignages métalliques font complètement défaut à l'est du méridien d'Alger, la partie orientale de l'Algérie et la Tunisie sont particulièrement riches en monuments funéraires qui, en Europe, sont antérieurs ou contemporains de l'Âge du bronze. Ces monuments sont rares au Maroc alors que se comptent par milliers les dolmens et hypogées d'Algérie orientale et de Tunisie. La répartition très particulière de ces monuments est un argument non négligeable en faveur de leur origine extérieure. Il existe manifestement une région mégalithique qui couvre la Tunisie centrale et l'Algérie orientale ; de part et d'autre s'étendent deux ailes littorales en Tunisie orientale et en Algérie jusqu'en Kabylie. Les derniers dolmens vers l'ouest sont ceux de Beni Messous*, à $20 \mathrm{~km}$ d'Alger. Nous avons déjà noté que c'est 
précisément dans ce secteur d'Algérie centrale que furent trouvés les derniers objets métalliques d'origine occidentale.

31 Ainsi les dolmens* nord-africains, qui sont de petite taille mais d'aspect tout à fait classique sont exactement dans le prolongement de ceux de Provence orientale, de Corse et de Sardaigne, constituant une sorte d'axe mégalithique méditerranéen. Mais alors que les dolmens d'Europe méridionale et des îles sont incontestablement d'âge très ancien, du Néolithique final-Chalcolithique, ceux d'Afrique du Nord n'ont livré aucun document qui permettent de leur reconnaître une aussi grande antiquité. Cependant il ne peut s'agir de monuments autochtones en raison de leur répartition particulière. Des analogies à travers la Méditerranée occidentale entre, par exemple, les Tombes de Géants de Sardaigne, les navetas de Minorque et les allées couvertes* de type kabyle, ne peuvent être le simple produit du hasard quand on reconnaît dans certains de ces monuments, aussi bien en Algérie (Aït Raouna) qu'en Sardaigne (Li Loghi) ou à Minorque (Es Tudons) une architecture intérieure à deux niveaux dans ces constructions qui sont parmi les plus spectaculaires des monuments funéraires allongés. Mais tandis que navetas et tombes de Géants se rapportent au chalcolithique et au premier Âge du bronze, les allées couvertes de Kabylie n'ont pas livré de céramiques antérieures au III siècle av. J.-C.!

Il nous faut donc imaginer que "l'idée mégalithique » a pénétré dans la partie orientale du Maghreb, au moment de l'extrême fin de son expansion et que cette région particulièrement conservatrice entretint longuement cette conception architecturale qui évolua sur place et donna naissance à des formes particulières. C'est le cas en Tunisie centrale, dans la région de Maktar où on peut suivre sur place le passage du dolmen simple au dolmen agrandi avec débordement volontaire de la dalle de couverture nécessitant l'implantation d'orthostates supplémentaires, parallèlement au grand côté de la cella, ainsi naît le dolmen à portique. Par ailleurs, le désir de rassembler plusieurs caveaux sous la même couverture mégalithique entraînait l'apparition des dolmens multiples. La conjonction des deux courants donna naissance aux grands monuments mégalithiques complexes à portique du type Ellez qui sont les plus belles réalisations du mouvement mégalithique au Sud de la Méditerranée. Ces monuments sont le témoin d'un culte funéraire exigeant. Les besoins de ce culte firent apparaître à leur tour de véritable chapelles qui s'insèrent entre les piliers du portique, une cour se développe en avant de celles-ci ; l'aboutissement de cette évolution est le monument de type Maktar qui présente une série de caveaux précédés de chapelles et de téménos mitoyens. A ce stade de l'évolution le monument funéraire mégalithique est devenu un sanctuaire.

On sait qu'il existe dans le nord de la Tunisie, et plus spécialement en Kroumirie et chez les Mogods, dans les petits massifs au nord de la Médjerda et au sud du cap Bon de petits hypogées cubiques creusés en flancs de rocher ou de falaise. Ils sont connus sous le nom de haouanet* ou de rhorfa ou encore de biban*. Ce mode de sépulture ne répond guère aux traditions funéraires des Carthaginois qui creusaient généralement très profondément leurs tombes, que ce soit les tombes à puits ou les caveaux construits dans une tranchée. Au contraire des nécropoles puniques voisines des principales villes, les haouanet sont dispersées dans les campagnes ou groupées à proximité de vieilles cités numides. Il arrive même que ces hypogées voisinent avec des dolmens et on a pu reconnaître des formes intermédiaires entre le hanout qui est creusé et le dolmen qui est construit. 
Il est vrai aussi que les analogies entre les haouanet et les tombes puniques à puits sont très fortes: même forme cubique ou à cul-de-four, même accès par une petite baie rectangulaire, même présence de niche cultuelle dans la paroi du fond et même décor géométrique. On pourrait facilement multiplier les preuves de la contemporanéité de nombreux haouanet et des tombes puniques. La cause est entendue, mais faut-il en conclure que les haouanet sont tous d'âge punique et qu'ils ont la même origine que les tombes puniques?

Armes et instruments en cuivre ou en bronze du Maghreb. Aucun objet métallique attribuable à l'Âge du bronze n'a été trouvé à l'est du méridien d'Alger.

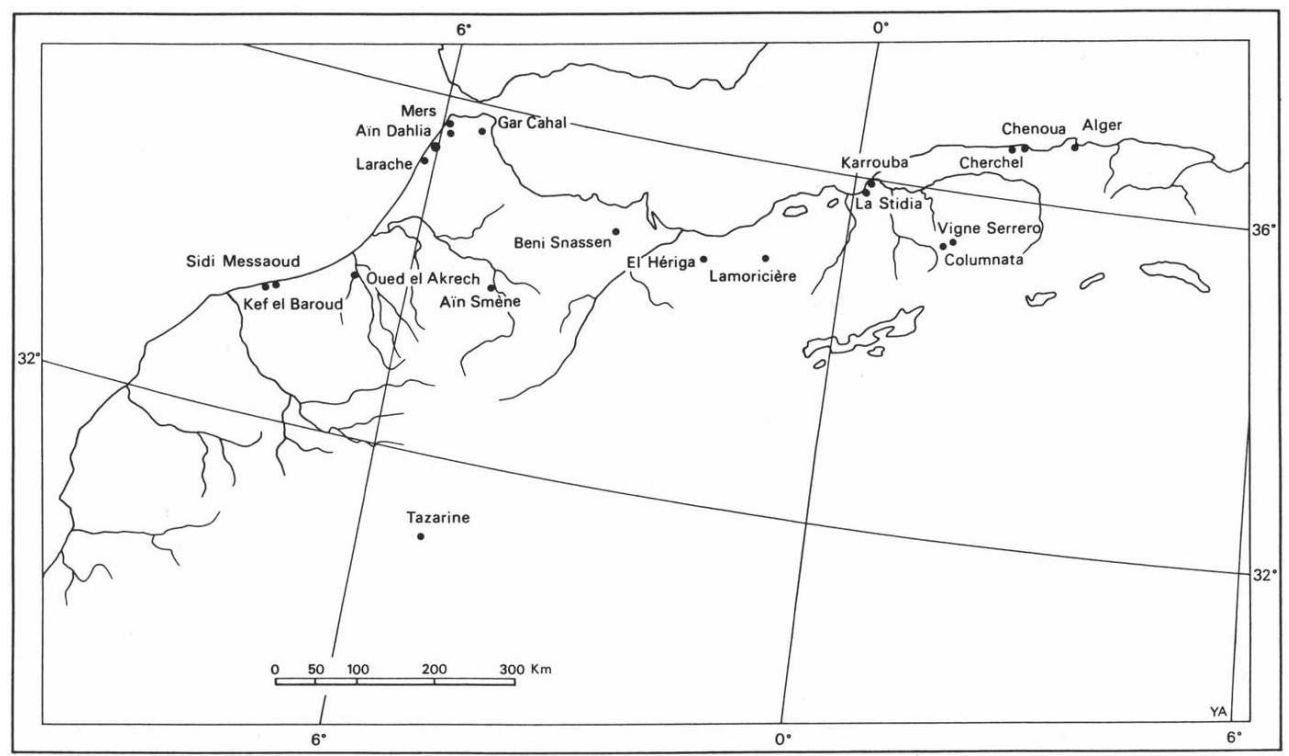

A vrai dire l'influence punique, qui est indiscutable, s'exerce sur un substrat qui ne peut être d'origine phénicienne. L'absence totale d'hypogées dans les zones les mieux punicisées (Basse Médjerda, environs de Carthage, Sahel, Syrte...) me paraît un argument non négligeable pour dissocier deux traditions funéraires d'origines distinctes. L'existence, en revanche d'hypogées de forme et de dimensions semblables en Sicile, en particulier dans la culture de Pantalica ou dans celle de Cassibile, invite à rechercher hors d'Afrique et hors des traditions phéniciennes l'origine des haouanet. Leur localisation dans le nord de la Tunisie et l'extrême nord-est de l'Algérie milite en faveur d'une origine méditerranéenne très ancienne dont la Sicile aurait pu être le relai ultime.

Il faut dire que la décoration des haouanet n'est pas toujours aussi punicisante qu'on le dit. Je retiendrai par exemple la scène sculptée en très bas relief sur une paroi d'un hanout du Jbel Mengoub qui représente un bovine aux très longues cornes devant lequel semble danser un personnage. Une scène célèbre du Kef el Blida se rapporte indiscutablement à une culture d'origine maritime, elle utilise des symboles qui ne paraisent guère puniques : le personnage principal brandit une hache bipenne et tient un bouclier à relief échancré en $\mathrm{V}$ datable du VII-VI ${ }^{e}$ siècle av. J.-C. Citons encore la place tenue par le taureau dans le décor de ces haouanet, en particulier le protomé de taureau en fort relief d'un hypogée du Jbel Sidi Zid qui détermine entre ses cornes une véritable niche. C'est aussi vers les plus anciennes traditions méditerranéennes qu'il faut se tourner pour expliquer la curieuse superposition de la colonne et de la niche cultuelle qui apparaît dans plusieurs haouanet du Cap Bon. Cette curieuse disposition si 
originale n'est connue, à mon sens, que dans la culture égéenne. Enfin comment ne pas faire de rapprochement entre la scène de danse devant le taureau du Jbel Mengoub et les jeux acrobatiques en présence du taureau dont l'art crétois a laissé d'admirables témoignages! Bien qu'ils soient le plus souvent d'âge punique, je suis convaincu que les haouanet qui se retrouvent identiques en Sicile, particulièrement dans le quart sud-est qui échappa toujours à la domination carthaginoise, tirent leurs origines d'un courant méditerranéen plus ancien et distinct de l'apport culturel phénicien. On a déjà associé l'apparition de ces petits hypogées dans le nord-est du Maghreb à l'introduction de la céramique peinte à décor géométrique qui a connu un brillant développement à Chypre, en Grèce archaïque, en Italie méridionale et en Sicile au cours de l'Âge du bronze et au début de l'Âge du fer. Parmi les multiples foyers de céramiques peintes susceptibles d'avoir diffusé leur technique vers l'Afrique du Nord, celui de Sicile semble devoir être retenu en priorité. Indépendamment de la proximité géographique et de l'existence dans les deux régions d'hypogées très semblables, il faut retenir l'importance et l'ancienneté des relations entre ces deux pays dès les temps néolithiques (introduction d'obsidienne en Tunisie). Les vases peints siciliens du style de Castelluccio présentent les mêmes motifs que ceux qui se sont maintenus jusqu'à nos jours sur les poteries peintes nord-africaines, de même des formes très particulières tels que les vases à filtre vertical se retrouvent de part et d'autre du détroit de Sicile, de même doit-on citer des détails techniques très caractéristiques parce que peu répandus, tel, par exemple le vernis à la résine appliquée sur les poteries siciliennes de Cassibile et sur celles de plusieurs régions de l'Algérie actuelle.

Bien que moins riche en objets métalliques que ses voisins européens, le Maghreb a participé au cours des $\mathrm{III}^{\mathrm{e}}$ et $\mathrm{II}^{\mathrm{e}}$ millénaires à l'intense développement des échanges, des relations maritimes et des courants de pensée, aussi bien le long des côtes atlantiques qu'à travers le bassin occidental de la Méditerranée.

\section{BIBLIOGRAPHIE}

ALMAGRO-BASCH M., «A proposito de unos objetos hachiformes representados en el arte rupestre del Sahara occidental », Munibe, t. 23, 1971, p. 25-35.

Atlas préhistorique de la Tunisie, f. 6 : La Goulette, par HARBI-RIAHI M., GRAGUEB A., CAMPS G., ZOUGHLAMI J., I.N.A., École franc, de Rome, 1987, fig. 64 et p. 52-60.

BATES O., The eastern Libyans, Londres, 1914.

BUCHET G., « Note préliminaire sur quelques sépultures anciennes du nord-ouest du Maroc », Bulletin de géographie historique et descriptive, 1907, p. 396-399.

CADENAT P., « Découverte d'une hache de bronze dans le commune mixte de Tiaret », Libyca, t. VI, 1956, p. 283-287.

CADENAT P., « Découvertes d'objets en bronze près de Tiaret », Bulletin de la Société préhistorique française, t. LVIII, 1961, p. 30-31. 
CAMPS G., « Les traces d'un Âge du bronze en Afrique du Nord », Rev. afric., t. 104, 1960, p. 31-55. CAMPS G., Aux origines de la Berbérie. Monuments et rites funéraiers protohistoriques, Paris, 1961, p. 403-408.

CAMPS G., Les civilisations préhistoriques de l'Afrique du Nord et du Sahara, Paris, Doin, 1974.

CAMPS G., « Protohistoire de l'Afrique du Nord. Questions de terminologie et de chronologie », REPPAL, t. 3, 1987, p. 43-70.

CAMPS G. et GIOT P.-R., « Un poignard chalcolithique au Cap Chenoua », Libyca, t. VIII, 1960, p.

261-278.

CAMPS G. et CADENAT P., « Nouvelle données sur le début de l'Âge des métaux en Afrique du Nord », Société d'Études et de Recherches préhistoriques, Les Eyzies, n 30, 1980, p. 40-51.

CHENORKIAN R., Les armes métalliques dans l'Art protohistorique de la Méditerranée occidentale, C.N.R.S., Paris, 1988.

DALLONI M., Géologie appliquée de l'Algérie, Alger, 1939.

GIOT P.-R. et SOUVILLE G., « La hache en bronze de l'Oued Akrech (Maroc) », II Congr. panaf. de Préhist., Alger, 1952, p. 221-239.

GSELL S., Histoire ancienne de l'Afrique du Nord, t. 1, p. 212.

JODIN A., « Les gisements de cuivre du Maroc et l'archéologie des métaux », Bull. d'Archéol. maroc., t. VI, 1966, p. 11-27.

JoDIN A., « L’Âge du bronze au Maroc : La nécropole mégalithique d'El Mriès (Oued Bou-Khalf, Tanger) », Bull. d'Archéol. maroc., t. V, 1964, p. 11-45.

MALHomme J., Corpus des gravues rupestres du Grand Atlas, Publ. du Serv. des Antiqu. du Maroc, Rabat, $1959,1961$.

PONSICH M., Recherches archéologiques à Tanger et dans sa région, Paris, 1970, p. 55 et sq., fig. 14, pl. 13. ROSENBERGER B., « Les anciennes exploitations minières et les anciens centres métallurgiques du Maroc », Rev. de Géogr. du Maroc, n 18, 1970, p. 59-102.

RUIZ-GALVEZ PRIEGO M., « Espada procedente de la Ria de Larache en el Museo de Berlin oeste », Homenage a Martin Almagro Basch, t. II, 1987, p. 63-68.

SOUVILLE G., « Recherches sur l'existence d'un Âge du bronze au Maroc », Atti del VI congresso internazionale delle Scienze preistoriche e protostoriche, 1962, p. 419-424.

SOUVILLE G., «Influences de la Péninsule ibérique sur les civilisations post-néolithiques du Maroc », Miscelanea en homenaje al abate H. Breuil, t. II, Barcelone, 1965, p. 409-422.

SOUVILLE G., « Une curieuse hache en bronze de la région de Beni Snassen », Bulletin d'archéologie marocaine, t. V, 1964, p. 319-323.

SOUVILLE G., « Témoignages sur l'Âge du bronze au Maghreb occidental », C.R.A.I.B.L., Janv.-mars 1987, p. 97-111.

TARRADELL M., « Noticia sobre la excavacion de Gar Cahal », Tamuda, t. II, 1954, p. 344-358.

WAILLY A. de, « Le site du Kef el Baroud (région de Ben Slimane) », Bulletin d'Archéologie marocaine, t. IX, 1973, p. 39-101. Id., " Le Kef el Baroud et l'ancienneté de l'introduction du cuivre au Maroc », ibid., t. X, 1976, p. 47-51. 
WENGLER L., « La grotte d'El Hariga (Maroc oriental) », Bulletin d'Archéologie marocaine, t. XV, 1983-1984, p. 81-90.

INDEX

Mots-clés : Protohistoire 